\title{
Bite-Block Relocation Error in Image-Guided Otologic Surgery
}

\author{
J. Michael Fitzpatrick ${ }^{1}$, Ramya Balachandran ${ }^{1}$, \\ and Robert F. Labadie ${ }^{2}$ \\ ${ }^{1}$ Department of Electrical Engineering and Computer Science \\ Vanderbilt University, Nashville TN 37212 \\ \{j.michael.fitzpatrick, ramya.balachandran\}@vanderbilt.edu \\ ${ }^{2}$ Department of Otolaryngology-Head and Neck Surgery \\ Vanderbilt University Medical Center, Nashville TN 37232 \\ robert. labadie@vanderbilt.edu
}

\begin{abstract}
Otologic surgery is undertaken to treat ailments of the ear including persistent infections, hearing loss, vertigo, and cancer. Typically performed on healthy patients in outpatient facilities, the application of image-guided surgery has been limited because accurate $(<1 \mathrm{~mm})$, non-invasive fiducial systems for otologic surgery are not available. We have developed such a system, which repeatably attaches to a subject via a dental bite block [1]. In a previous report, ex-vivo validation indicated that mean target registration error \pm standard deviation for 234 targets within the surgical field was $0.73 \pm 0.23 \mathrm{~mm}$ with a root mean square of $0.77 \mathrm{~mm}[2,3]$. In an effort to further understand the limitations of our system, we undertook, and report herein, a detailed error analysis to determine what portion of the error is attributable to the inaccuracies in relocation fit of the fiducial frame to a subject's dentition. Our analysis shows that the root-mean-square error due to relocation alone is approximately 0.66 $\mathrm{mm}$, while that due to fiducial registration is approximately $0.30 \mathrm{~mm}$.
\end{abstract}

\section{Introduction}

Image-Guided Surgery (IGS) has found widespread use in neurosurgery where boneimplanted fiducial markers are used. Such invasive fiducial systems are tolerated by patients with life-threatening diseases such as malignant brain tumors. Ear surgery shares many of the characteristics of neurosurgery, i.e., microscopic surgery performed in the region of the cranium, but differs in that the majority of cases are for non-malignant processes. As such, invasive fiducial marker systems are unacceptable. Non-invasive fiducial systems, such as those based on skin-affixed fiducial markers and laser contouring, have not met the requirement of submillimetric accuracy.

These seemingly paradoxical design criteria, high accuracy and non-invasiveness, can be overcome by means of fiducial frame systems that attach to the teeth [1]. In this paper we report on a fiducial system based on a dental bite-block with an attached rigid frame containing 14 fiducial markers whose configuration surrounds the surgical 
field of interest, the ear. Previous studies have confirmed submillimetric accuracy $[2,3]$. Figure 1 shows the system attached to a human skull. Because the least reproducible component of our system is the coupling of the fiducial frame to the subject's dentition (Figure 1b), we set out to analyze its contribution to target registration error. The analysis is based on tests with three human skulls.

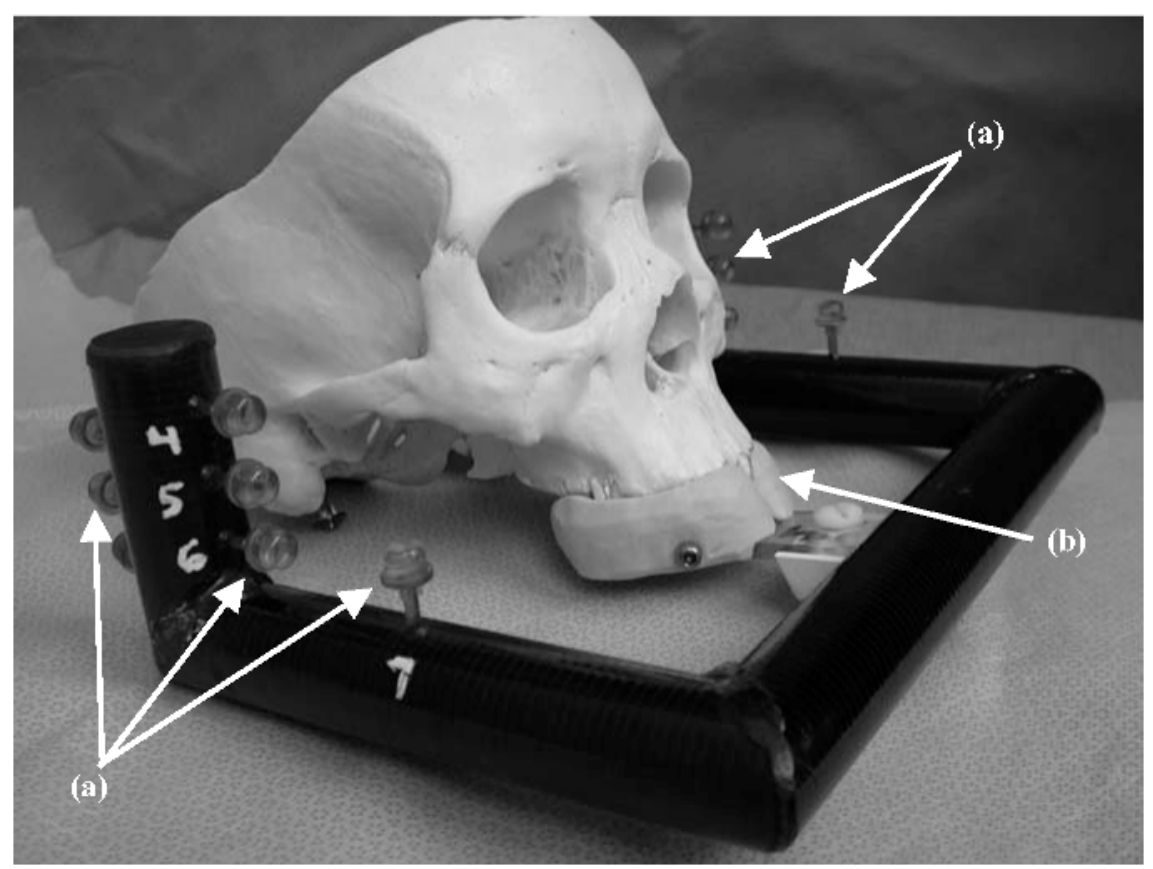

Fig. 1. Fiducial frame system. (a) Fiducials attached to frame. (b) Dental bite block by means of which the frame is attached to the upper dentition of a cadaveric skull.

\section{Methods}

The three skulls had their temporal bones removed and were retrofitted with an independent registration system, which we call the "target system", attached directly to bone within the region of the temporal bone, the bone encasing the left ear. The target system consisted of a disk with 12 markers placed in a centered cross-hair pattern in the vicinity of the surgical field. This system is named the "target system" because it is located in the vicinity of the surgical targets. Each skull was also affixed with the fiducial system coupled to the skull via a dental bite block [4]. Clinically applicable CT scans (slice thickness $=0.5 \mathrm{~mm}$ ) were obtained, and the centroids of all markers (i.e., both the fiducial-system markers and target-system markers) were identified using previously described algorithms [5]. Next, each skull was transported 
to the laboratory where the bite block was removed from the skull and reattached. The physical location of all markers were then determined using a 3-D localization systems based on a commercially available infrared tracking system, the active Polaris system, manufactured by Northern Digital, Inc. (Waterloo, Ontario, Canada) including a coordinate reference frame (not shown) attached to the fiducial frame and a tracked probe that is brought into contact with the markers (also not shown), all of which is driven by the Voyager image analysis and display system manufactured by Z-Kat, Inc. (Hollywood, FL). Three sets of physical space acquisitions were obtained for each skull. In each case the probe was oriented so as to keep its infrared emitters aimed at the Polaris. The skull with attached frame was re-oriented during localization as needed but with the attached coordinate reference frame kept continuously aimed at the Polaris.

Rigid registration between physical and radiographic space was performed using all 14 fiducial markers and also using nine subsets containing six of the 14 markers on the fiducial system such that the centroid of the six markers varied for each of the subsets. Registration was performed using singular-value decomposition of the crosscovariance matrix to minimize fiducial registration error (FRE), which is the rootmean-square distance between corresponding fiducials after registration [6]. A set of 12 target positions was chosen in the surgical field of the left ear. For each target position, target registration error (TRE) was measured experimentally.

The experimental measurement of TRE was accomplished by comparing two registrations: Registration 1, which is accomplished by aligning either all fiducialsystem localizations or a subset of 6 localizations in the two spaces (CT and physical), and Registration 2, which is accomplished by matching the set of 12 target-system localizations in the two spaces. The comparison of the two registrations is accomplished by applying the two transformations in turn to each of the target positions in image space and measuring the disparity between their transformed positions in physical space. Each transformation will suffer error due to fiducial localization error (FLE). Since these errors are independent, the error that we measure will be the vector sum of the errors caused by the two systems.

In addition to the error caused by FLE, repositioning of the bite block relative to the teeth, and hence to the skull, will cause additional error. It is that error that we wish to assess. While it is not possible experimentally to separate the components of error, due to FLE and due to movement of the frame, it is possible to estimate the former error by means of theoretical considerations. The error due to frame movement also adds vectorially, and because it is unrelated to the error due to FLE, its error can be expected to be uncorrelated. As a result, we can expect that for each target position, the errors will add in quadrature, as stated in Eq. (1).

$$
\left\langle\mathrm{TRE}_{\text {total }}^{2}\right\rangle=\left\langle\mathrm{TRE}_{\mathrm{FLE}}^{2}\right\rangle+\left\langle\mathrm{TRE}_{\text {frame }}^{2}\right\rangle,
$$

where $\langle\cdot\rangle$ means expected value. We assess the frame repositioning error by estimating $\left\langle\mathrm{TRE}_{\text {frame }}^{2}\right\rangle$. To do that, we employ the formulation given in [6] to 
calculate the theoretical value of $\left\langle\mathrm{TRE}_{\mathrm{FLE}}^{2}\right\rangle$. We then repeatedly measure $\left|\mathrm{TRE}_{\text {total }}\right|$ experimentally and average to get an estimate of the left side of Eq. (1). Finally, we estimate $\left\langle\mathrm{TRE}_{\text {frame }}^{2}\right\rangle$ by subtracting:

$$
\left\langle\mathrm{TRE}_{\text {frame }}^{2}\right\rangle=\overline{\left|\mathrm{TRE}_{\text {total }}^{2}\right|}-\left\langle\mathrm{TRE}_{\mathrm{FLE}}^{2}\right\rangle
$$

where the horizontal bar means "average of experimentally measured values".

The calculation of $\left\langle\mathrm{TRE}_{\mathrm{FLE}}^{2}\right\rangle$ must take into account the errors arising from both Registration 1 and Registration 2. The difference between these two calculations arises from the difference between the configurations of the markers in the fiducial system and in the target system. Since there are no markers in common between these two systems, their target errors are independent. Thus,

$$
\left\langle\mathrm{TRE}_{\mathrm{FLE}}^{2}\right\rangle=\left\langle\mathrm{TRE}_{1}^{2}\right\rangle+\left\langle\mathrm{TRE}_{2}^{2}\right\rangle
$$

where the subscripts 1 and 2 refer to Registrations 1 and 2, respectively. The calculation of each of the terms on the right is based on the equation [6,7],

$$
\left\langle\operatorname{TRE}^{2}(\mathbf{r})\right\rangle=\frac{\left\langle\operatorname{FLE}^{2}\right\rangle}{N}\left(1+\frac{1}{3} \sum_{k=1}^{3} \frac{d_{k}^{2}}{f_{k}^{2}}\right),
$$

where $\mathbf{r}$ is the target position, $\left\langle\mathrm{FLE}^{2}\right\rangle$ is the expected value of fiducial localization error, $N$ is the number of fiducials used in the registration (six for Registration 1 and twelve for Registration 2), $d_{k}$ is the distance of $\mathbf{r}$ from the $k$ th principal axis of the fiducial set, and $f_{k}$ is the root-mean square distance of the fiducials themselves from their $k$ th principal axis. The value of $\left\langle\mathrm{FLE}^{2}\right\rangle$ is estimated by using the relationship $[6,7]$,

$$
\left\langle\mathrm{FRE}^{2}\right\rangle=(1-2 / N)\left\langle\mathrm{FLE}^{2}\right\rangle
$$

where $N$ is the number of fiducials. For these experiments, Eq. (5) was applied to the fiducial system for each subset of markers (Registration 1) with $N$ equal to 6 and to the target system with $N$ equal 12 (Registration 2 ). 


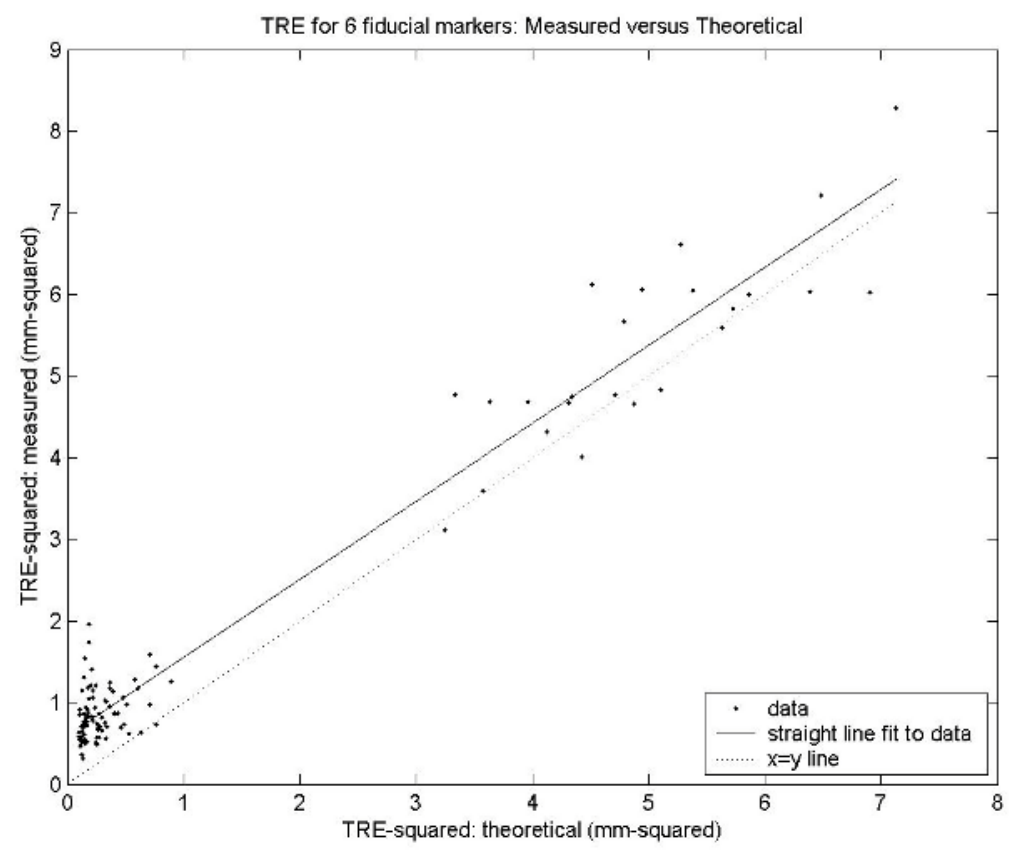

Fig. 2. TRE: Measured versus theoretical for combinations of 6 fiducials

\section{Results}

Figure 2 shows a plot of $\overline{\left|\mathrm{TRE}_{\text {total }}^{2}\right|}$ versus $\left\langle\mathrm{TRE}_{\mathrm{FLE}}^{2}\right\rangle$. There are 108 plotted points, each point representing one of the 12 target positions and one of the nine subsets of markers from the fiducial system (in each case all 12 markers were used for the target system). The value of $\overline{\left|\mathrm{TRE}_{\text {total }}^{2}\right|}$ was gotten in each case by averaging the squared values of TRE over three measurements on three skulls (9 independent measurements). The value of $\left\langle\mathrm{TRE}_{\mathrm{FLE}}^{2}\right\rangle$ was gotten in each case by averaging the values calculated from Eqs. (3) and (4) for the three skulls. The value of $\left\langle\mathrm{FLE}^{2}\right\rangle$ used in Eq. (4) is calculated from Eq. (5).

The solid line is a least-squares regression line. Its slope is 0.95 and its intercept is $0.60 \mathrm{~mm}^{2} ; R^{2}=0.96$. The dotted line (slope $=1$, intercept $=0$ ) is the ideal, which would be expected if the only contribution to error were that arising from FLE (with no error arising from the relocation error of the bite block). The subsets of the markers of the fiducial system include two that were purposefully chosen to be substandard arrangements for the target positions. These arrangements are 
substandard because in each case all 6 markers are located on the right side of the head, while the target positions are on the left side. As a result, the centroid of the marker configuration is far from the target positions. The 24 points at the upper right of the plot were produced by these two combinations. The remaining points, which are clustered at the lower left, were produced by arrangements that included at least one marker on each side of the skull.

The smaller errors of the points at the lower left of Figure 2, as compared with the others show that it is important to position markers used as fiducials for registration such that the centroid of their configuration is near the target region. This point has been emphasized before as has the importance of including a large number of markers [8]. For these reasons, the frame includes 14 markers with 7 positioned on each side of the head. Using all 14 fiducial markers, we observed for $\overline{\left|\mathrm{TRE}_{\text {total }}^{2}\right|}$ a value of 0.53 $\mathrm{mm}^{2}$ over all three skulls, all three trials for each skull, and all 12 targets for each trial. Using Eqs. (3) and (4), we calculated for $\left\langle\mathrm{TRE}_{\mathrm{FLE}}^{2}\right\rangle$ a value of $0.09 \mathrm{~mm}^{2}$ for all three skulls and all 12 targets. Using these two values in Eq. (2), we calculated $\left\langle\mathrm{TRE}_{\text {frame }}^{2}\right\rangle$ to be $0.44 \mathrm{~mm}^{2}$. Taking square roots, we find the root-mean-square values given in Table 1 .

Table 1. Root-Mean-Square TRE Values

\begin{tabular}{|c|l|}
\hline TRE $_{\text {total }}$ & $0.73 \mathrm{~mm}$ \\
\hline $\mathrm{TRE}_{\text {FLE }}$ & $0.30 \mathrm{~mm}$ \\
\hline $\mathrm{TRE}_{\text {frame }}$ & $0.66 \mathrm{~mm}$ \\
\hline
\end{tabular}

The root-mean-square FRE for all skulls and all trials was also calculated and found to be $0.69 \mathrm{~mm}$; the root-mean-square FLE $=0.74 \mathrm{~mm}$. Contributions to FLE error come from the localization of the markers in the CT images and from the error in physical localization. The latter error comprises two components that combine in quadrature, as described in [9]: the error in tracking the probe and the error in tracking the coordinate reference frame, which is situated at an average distance of $190 \mathrm{~mm}$ from the targets.

\section{Discussion}

Other groups have also reported registration errors based on bite-blocks that mount to the patient's maxillary dentition $[4,10,11]$, but none of them has employed theoretical calculations to determine the contribution to error caused by the error in the application of the bite block. 
In order to employ the theoretical estimation of $\mathrm{TRE}_{\mathrm{FLE}}$ in our analysis, it is important to justify its validity for this experiment by demonstrating agreement between measured TRE and theoretical TRE. Figure 2, which is a plot similar to that shown recently by West and Maurer [9], provides this demonstration by including both excellent and poor fiducial combinations so as to provide a wide range of TREs. The obvious correlation of the measured TRE with the theoretical TRE is a strong indication that the theoretical calculation is correct for this application. That correlation provides our justification for using the theoretical value in Eq. (2) to estimate the relocation error. The roughly constant vertical disparity between the least-squares fit line (solid) and the ideal line (dotted) is to be expected because of the relocation error of the dental bite-block. (It is in fact this error that is the major subject of the paper.) The square-root of the intercept of the solid line in Figure 2, which should be approximately equal to $\mathrm{TRE}_{\text {frame }}$, is $0.77 \mathrm{~mm}$, which indicates that the frame error may be slightly higher than the value given in Table 1.

\section{Conclusion}

These results demonstrate that for this system most of the target registration error is caused by error in the repositioning of the dental bite-block. However, the results also demonstrate that, thanks to the small size of this error and the considerably smaller size of the error due to fiducial localization, submillimetric target registration error is achievable with this system, when all fiducial markers are used. The clinical application of this system is currently being validated intraoperatively.

\section{References}

1. Labadie RF, Fenlon M, Devikalp H, et al. Image-guided otologic surgery. Computer Assisted Radiology and Congress and Exhibition (eds: Lemke HU, Vannier MW, Inamura K, Farman AG, Doi K, Reiber JHC) pp. 627-32. Elsevier Science, Amsterdam, The Netherlands, 2003.

2. Labadie RF, Shah RJ, Harris SS, et al. Image - Guided Otologic Surgery: Submillimeter Accuracy within the Temporal Bone, Oto-HNS (in submission).

3. Labadie RF, Shah RJ, Harris SS, et al. Submillimetric Target-Registration Error using a Novel, Non-Invasive Fiducial System (the EarMark ${ }^{\mathrm{TM}}$ ) for Image Guided OtologicSurgery, JCAS (in submission).

4. Fenlon MR, Jusczyzck AS, Edwards PJ, and King AP. Locking acrylic resin dental stent for image guided surgery. J of Prosthet Dent;83:482-5, 2000.

5. Wang MY, Maurer Jr. CR, Fitzpatrick JM, and Maciunas RJ. An automatic technique for finding and localizing externally attached markers in CT and MR volume images of the head. IEEE Trans Biomed Eng;43:627-37, 1996.

6. Fitzpatrick JM, Hill DLG, and Maurer CR. Registration. Medical Image Processing, Volume II of the Handbook of Medical Imaging, M. Sonka and J. M. Fitzpatrick, ed., SPIE Press. 447-513, 2000. 
7. Fitzpatrick JM, West JM, Maurer Jr. CR. Predicting error in rigid-body, point-based registration. IEEE Trans Med Imaging 17, 694-702, 1998.

8. J. B. West*, J. M. Fitzpatrick, S. Toms, C. R. Maurer, Jr.*, R. J. Maciunas, "Fiducial point placement and the accuracy of point-based, rigid-body registration", Neurosurgery 48, 810-817, 2001.

9. West JB and Maurer CR. Designing optically tracked instruments for image-guided surgery. IEEE Trans. Med. Imaging 23: 533-545, 2004.

10. Bale RJ, Burtscher J, Eisner W, et al. Computer-assisted neurosurgery by using a noninvasive vacuum-affixed dental cast that acts as a reference base: another step toward a unified approach in the treatment of brain tumors. J Neurosurg, 93: 208-13, 2000.

11. Meeks SL, Bova FJ, Wagner TH, et cl. Image localization for frameless stereotactic radiotherapy. Int J Radiat Oncol Biol Phys;4,6:1291-9, 2000. 